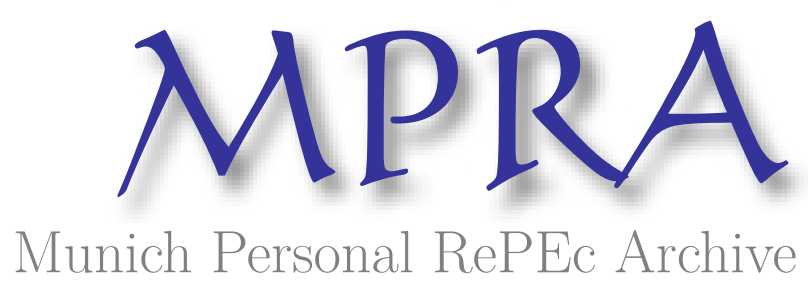

\title{
A social proximity explanation of the reluctance to assimilate
}

Fan, C. Simon and Stark, Oded

2007

Online at https://mpra.ub.uni-muenchen.de/30940/

MPRA Paper No. 30940, posted 17 May 2011 12:55 UTC 


\title{
A Social Proximity Explanation of the Reluctance to Assimilate
}

\author{
C. Simon Fan* and Oded Stark**
}

* C. Simon Fan, Department of Economics, Lingnan University, 8 Castle Peak Road, Tuen Mun, New Territories, Hong Kong. E-mail: fansimon@ln.edu.hk.

** Oded Stark, Universities of Klagenfurt, Bonn, and Vienna; Warsaw University; ESCE Economic and Social Research Center, Cologne and Eisenstadt. Mailing address: University of Klagenfurt, Universitaetsstrasse 65-67, A-9020 Klagenfurt, Austria.

E-mail: ostark@uni-bonn.de.

We are indebted to Donald Cox, Ewa Kepinska, Maja Micevska, and Anna Zylicz for enlightening Comments. 


\begin{abstract}
Quite often, migrants appear to exert little effort to absorb the mainstream culture and to learn the language of their host society, even though the economic returns (increased productivity and enhanced earnings) to assimilation are high. We show that when interpersonal comparisons affect individuals' wellbeing and when a more intensive assimilation results in migrants' comparing themselves more with the richer natives and less with fellow migrants, then the effort extended to assimilate will be muted.
\end{abstract}

Keywords: Migrants' behavior; The effort to assimilate; Income gains; Social proximity; Interpersonal comparisons; Deprivation losses

JEL Classification: F22; J15; J24; J61; O15; Z10 


\section{INTRODUCTION}

Common culture and common language facilitate communication and interaction between individuals. Consequently, migrants' assimilation into the mainstream culture of their host country is likely to increase their productivity and earnings. There is considerable empirical research on the relationship between migrants' assimilation and their earnings, and this literature often finds that migrants exert little effort to absorb the mainstream culture and language, even though the economic returns to assimilation are high. ${ }^{1}$ For example, Lazear (1999, p. S96) concludes: 'Multiculturalism .... seems to be on the rise in the United States.... In 1900, 85 percent of immigrants were fluent in English. Surprisingly, in 1990 the fluency rate among immigrants was only 68 percent, despite dramatic improvements in communication during the century.' This is puzzling. In a recent compilation (Friedman and Randeria, 2004) the failure of migrants to integrate, conform, and assimilate is portrayed quite extensively. The contributors to the compilation express concern and explore possible means of transforming migrants into 'ethnics' and 'nationals.' However, the proposed policies do not allow that migrants could optimally elect not to assimilate.

In the received literature, rigorous theoretical analyses of assimilation are rare, which contrasts with the related empirical literature. ${ }^{2}$ A notable exception is Lazear, who argues that assimilation may not occur when the migrants' own community is large and when it is costly for them to learn the host country's culture and language. In this paper we present a new model of assimilation that draws on the literature of relative deprivation and interpersonal comparisons.

Our model assumes that, to an extent, the reference groups of an individual are

1. See, for example, Borjas (1995), Chiswick (2005), and Cutler, Glaeser, and Vigdor (2005). Borjas, Bronars, and Trejo (1992) provide estimates of the average hourly earnings of young international migrants in the United States. Looking at a cross-section of individuals between the ages of 21 and 29 from the 1980 U.S. Census, they find sizable returns to speaking English.

2. While occasionally the substantial empirical studies point to interesting theoretical arguments and explanations (cf. Chiswick, 2005), these arguments and explanations are mostly not formalized rigorously. 
chosen endogenously. ${ }^{3}$ This assumption is in line with the recent literature that explores the social and psychological repercussions of individual behavior, cf. Akerlof's (1997) concept of 'social distance,' Agarwal and Vercelli (2005), Austen-Smith and Fryer (2005) and the research reviewed therein. For example, Akerlof (p. 1010) writes: 'I shall let individuals occupy different locations in social space. Social interaction ... will increase with proximity in this space. Current social location is acquired and dependent on the [individual's] decision ... .' Drawing on this literature, we posit that the more effort a migrant exerts in assimilating into the mainstream culture, the closer he will be in social space to the natives and the farther away he will be from his fellow migrants. We show that when interpersonal comparisons affect individuals' wellbeing and when a more intensive assimilation results in migrants' comparing themselves more with the richer natives and less with their fellow migrants, then the effort extended to assimilate is muted. Regarding the empirical content of our model we predict, inter alia, that the larger the income gap between the natives and the migrants, the weaker the effort to assimilate.

\section{ANALYSIS}

Consider a set of migrants who originate from a poor country and who are in a rich country. To focus on essentials, we assume that in all relevant respects the migrants are identical. ${ }^{4}$ Each migrant decides how much effort to exert in order to assimilate himself into the mainstream culture of the host country. If a migrant is better assimilated into the mainstream culture, he will earn a higher income. However, upon assimilating more, he will become closer to the (rich) natives when making interpersonal comparisons. Formally, let a migrant's utility function be

$$
u=Y(x)-R D-C(x)
$$

3. To the best of our knowledge, the current paper provides a rare model of the endogenous formation of reference groups. Hence, the paper adds to the literature on relative deprivation.

4. However, even if there is some heterogeneity within the group of migrants, the results that follow will go through. What is critical for our argument is the persistent prevalence of an earnings gap between the natives and the migrants, not the homogeneity of the group of migrants. 


$$
Y^{\prime}(x)>0, Y^{\prime \prime}(x)<0 ; \quad C^{\prime}(x)>0, C^{\prime \prime}(x)>0
$$

where " $Y$ " denotes the migrant's income, " $R D$ " denotes the relative deprivation of the migrant which arises from interpersonal comparisons, ${ }^{6}$ " $x$ " denotes the migrant's effort to assimilate, and " $C$ " is the cost of extending this effort. We assume that $\lim _{x \rightarrow 0} Y^{\prime}(x)=\infty$, namely that the Inada condition holds, thereby ensuring that in the analysis that follows the solutions of $x$ are interior.

The relative deprivation of an individual is defined as follows

$$
\begin{aligned}
& F(I, R) \\
& \frac{\partial F}{\partial I}<0, \frac{\partial F}{\partial R}>0
\end{aligned}
$$

where " $I$ " is the individual's own income, and " $R$ " is the average income of his reference group. We assume that a migrant's set of reference groups consists of the natives and of his fellow migrants. The average income of the natives is denoted by ' $Z$ ' and is assumed to be higher than the migrant's earnings no matter how hard the migrant tries to assimilate into the mainstream culture; the average income of the other migrants is denoted by $\bar{Y}$ which, in turn, is determined by other migrants' average effort to assimilate into the mainstream culture, $\bar{x}$.

We assume that the more a migrant assimilates into the mainstream culture, the more he will compare himself with the natives and the less he will compare himself with his fellow migrants. The relative weight of his being affiliated with the natives and thereby comparing himself with them is $p(0 \leq p \leq 1)$, and the corresponding weight

5. We can also interpret $Y$ more generally to encompass the utility from "consuming" the mainstream culture of the host country.

6. The idea that relative income impinges on welfare dates back at least to Veblen (1924). For pioneering and recent writings on the analytics of relative deprivation see, respectively, Yitzhaki (1979), and Walker and Smith (2002). For writings on relative deprivation and migration see Stark (1993), and Stark and Wang (2005). For recent empirical studies that demonstrate the importance of relative deprivation see, for example, Eibner and Evans (2005), and Luttmer (2005). 
pertaining to the migrant's fellow migrants is $1-p$. Formally, we define

$$
\begin{aligned}
& p \equiv p(x) \\
& p^{\prime}(x)>0
\end{aligned}
$$

Then, we can express the migrant's relative deprivation, " $R D$ ", as a weighted sum

$$
R D=p(x) F[Y(x), Z]+[1-p(x)] F[Y(x), \bar{Y}]
$$

Inserting (4) into (1), we get

$$
u=Y(x)-p(x) F[Y(x), Z]-[1-p(x)] F[Y(x), \bar{Y}]-C(x)
$$

Note that a standard utility formulation captures the tension between the unpleasant exertion of effort aimed at acquiring productive "tools," and the consequent pleasing derivation of income (the last and first terms on the right hand-side of (5), respectively). The added RD terms (the middle terms on the right hand-side of (5)) introduce an additional dimension of tension: the effort to acquire productive "tools" results in a reduction in the displeasure that arises from a relatively low income within both reference groups, yet it increases the weight that is accorded to the natives as a reference group within which comparisons result in substantial discontent. ${ }^{7,8}$

In writing $p$ as $p(x)$, we are making two important assumptions: first, that the set of reference groups is determined endogenously (by $x$ ) and that it is not predetermined on the basis of ethnicity, origin, and so on. And second, that the exertion of the assimilation effort and the proximity to the natives are intertwined. Hence, for example, it is not

7. Recall the assumption that even if a migrant tries very hard to assimilate into the mainstream culture, he will still earn an income that is lower than that of the natives.

8. For simplicity's sake, we assume away possible repercussions of the selection of the assimilation effort by the migrants upon the relative deprivation of the natives. However, even if such repercussions were to be considered then, if the relative deprivation of the natives is defined in a manner akin to that of the migrants, and if the income gap between the natives and the migrants remains large - the assimilation effort of the migrants notwithstanding, the relative deprivation of the natives will not be affected by the migrants' choice of the assimilation effort. 
possible to choose to exert a high level of assimilation effort while at the same time to exclude the natives as a reference group.

The migrant's optimization problem is to choose $x$ such as to bring his utility in (5) to a maximum (given his belief about the value of $\bar{x}$ and hence about the value of $\bar{Y})$. The first-order condition for a maximum of (5) is

$$
\begin{aligned}
& Y^{\prime}(x)-p^{\prime}(x) F[Y(x), Z]-p(x) Y^{\prime}(x) F_{1}[Y(x), Z] \\
& +p^{\prime}(x) F[Y(x), \bar{Y}]-[1-p(x)] Y^{\prime}(x) F_{1}[Y(x), \bar{Y}]-C^{\prime}(x)=0
\end{aligned}
$$

Since all migrants are identical, in equilibrium we must have that $x=\bar{x}$ and hence

$$
Y(x)=\bar{Y}
$$

Naturally, we assume that

$$
F[Y(x), Y(x)]=0
$$

When migrants are identical and each migrant extends the same level of effort as any other migrant, then there is no difference in incomes within the group of migrants and hence no relative deprivation arises from intra-group comparisons.

Inserting (7) and (8) into (6), we get

$$
\begin{aligned}
& Y^{\prime}(x)-p^{\prime}(x) F[Y(x), Z]-p(x) Y^{\prime}(x) F_{1}[Y(x), Z] \\
& -[1-p(x)] Y^{\prime}(x) F_{1}[Y(x), Y(x)]-C^{\prime}(x)=0
\end{aligned}
$$

Clearly, if the solution to (9) is unique, then there exists a unique Nash equilibrium for the level of effort that a migrant exerts in assimilating into the mainstream culture. ${ }^{9}$ If the

9. A sufficient condition for the existence of a unique Nash equilibrium is that the derivative of the left hand-side of (9) with respect to $x$ is negative. 
solution to (9) is not unique, then there exist multiple Nash equilibria.

The analysis yields an interesting implication. Suppose that the solution to (9) is not unique, so that there exist multiple Nash equilibria. Then, for two similar (in all relevant respects) groups of migrants in a host country with a given mainstream culture, members of one group may exert more effort to assimilate to the mainstream culture than members of the other group.

Had interpersonal comparisons not been considered - a special case of (1) - the migrant's utility function would have become

$$
u=Y(x)-C(x)
$$

The first-order condition for a maximum of (10) is

$$
Y^{\prime}(x)-C^{\prime}(x)=0
$$

We denote the solution to (11) by $x_{1}^{*}$.

We now denote the (largest) solution to (9) by $x_{2}^{*}$. Then, from comparing (11) with (9), we derive the following proposition which provides a condition under which the concern for interpersonal comparisons mutes the optimal assimilation effort.

Proposition 1: $x_{2}^{*}<x_{1}^{*}$ if

$$
\begin{aligned}
& p^{\prime}\left(x_{2}^{*}\right) F\left[Y\left(x_{2}^{*}\right), Z\right]+p\left(x_{2}^{*}\right) Y^{\prime}\left(x_{2}^{*}\right) F_{1}\left[Y\left(x_{2}^{*}\right), Z\right] \\
& +\left[1-p\left(x_{2}^{*}\right)\right] Y^{\prime}\left(x_{2}^{*}\right) F_{1}\left[Y\left(x_{2}^{*}\right), Y\left(x_{2}^{*}\right)\right]>0
\end{aligned}
$$

Proof. We prove the claim by contradiction. Suppose otherwise, namely that $x_{2}^{*} \geq x_{1}^{*}$. From (11) we have 


$$
Y^{\prime}\left(x_{1}^{*}\right)-C^{\prime}\left(x_{1}^{*}\right)=0
$$

Since $Y^{\prime \prime}(x)-C^{\prime \prime}(x)<0$ then from (13) and $x_{2}^{*} \geq x_{1}^{*}$ we have

$$
Y^{\prime}\left(x_{2}^{*}\right)-C^{\prime}\left(x_{2}^{*}\right) \leq 0
$$

Thus, we have that

$$
\begin{aligned}
& Y^{\prime}\left(x_{2}^{*}\right)-p^{\prime}\left(x_{2}^{*}\right) F\left[Y\left(x_{2}^{*}\right), Z\right]-p\left(x_{2}^{*}\right) Y^{\prime}\left(x_{2}^{*}\right) F_{1}\left[Y\left(x_{2}^{*}\right), Z\right] \\
& -\left[1-p\left(x_{2}^{*}\right)\right] Y^{\prime}\left(x_{2}^{*}\right) F_{1}\left[Y\left(x_{2}^{*}\right), Y\left(x_{2}^{*}\right)\right]-C^{\prime}\left(x_{2}^{*}\right) \\
& =\left[Y^{\prime}\left(x_{2}^{*}\right)-C^{\prime}\left(x_{2}^{*}\right)\right]-\left\{p^{\prime}\left(x_{2}^{*}\right) F\left[Y\left(x_{2}^{*}\right), Z\right]+p\left(x_{2}^{*}\right) Y^{\prime}\left(x_{2}^{*}\right) F_{1}\left[Y\left(x_{2}^{*}\right), Z\right]\right. \\
& \left.+\left[1-p\left(x_{2}^{*}\right)\right] Y^{\prime}\left(x_{2}^{*}\right) F_{1}\left[Y\left(x_{2}^{*}\right), Y\left(x_{2}^{*}\right)\right]\right\} \\
& <0
\end{aligned}
$$

which contradicts (9). Thus, we have proved the proposition.

We can rewrite (12) as

$$
p^{\prime}\left(x_{2}^{*}\right) F\left[Y\left(x_{2}^{*}\right), Z\right]>-\left\{p\left(x_{2}^{*}\right) F_{1}\left[Y\left(x_{2}^{*}\right), Z\right]+\left[1-p\left(x_{2}^{*}\right)\right] F_{1}\left[Y\left(x_{2}^{*}\right), Y\left(x_{2}^{*}\right)\right]\right\} Y^{\prime}\left(x_{2}^{*}\right)
$$

Clearly, the larger $p^{\prime}\left(x_{2}^{*}\right)$ and $F\left[Y\left(x_{2}^{*}\right), Z\right]$, the smaller the absolute values of $F_{1}\left[Y\left(x_{2}^{*}\right), Z\right]$ and $F_{1}\left[Y\left(x_{2}^{*}\right), Y\left(x_{2}^{*}\right)\right]$, and the smaller $Y^{\prime}\left(x_{2}^{*}\right)$, the more likely will inequality (16) (and hence inequality (12)) be satisfied.

Note that the left-hand side of (16) represents the marginal (dis)utility cost, due to an increase in $x$, that arises from the sensing of relative deprivation upon a comparison with the natives, whereas the right-hand side of (16) represents the marginal utility benefit, due to an increase in $x$, of increasing a migrant's standing among other migrants and of narrowing the income gap between him and the natives. Thus, inequality (16) (and 
hence inequality (12)) is more likely to be satisfied if more intensive assimilation results in a relatively high cost - from a migrant's comparing himself more with the richer natives - and a relatively low benefit - from his increasing his standing among fellow migrants. Hence, under the stated condition, the proposition implies that the choice of social proximity to reference groups and interpersonal comparisons entails migrants' having a weak incentive to accumulate the skills that would enhance their productivity.

Our analysis yields interesting empirical implications. For example, it is observed that migrants' segregation in the United States has increased since 1970, and it has been suggested that the increased segregation is due to greater racial dissimilarity with the American majority population (Cutler, Glaeser, and Vigdor, 2005). An explanation of the said segregation trend is that 'Greater linguistic differences between immigrants' native tongues and English has created a greater demand for enclave communities that offer opportunities to reduce communication costs'(Cutler, Glaeser, and Vigdor, 2005, p.29). But why is it that the linguistic differences were not narrowed by means of an assimilation effort (language acquisition)? Our analysis suggests that it is the rising income divide between the native population and the migrants that could account for the growing reluctance to assimilate and consequently for the increased segregation.

This analysis is also related to Akerlof and Kranton (2000), who inquire how identity, that is, a person's sense of self, affects economic outcomes. In particular, they propose a utility function in which identity is associated with different social categories, and they proceed to analyze how people in these categories behave. The analysis in our paper examines how an important dimension of an individual's identity can be determined endogenously: we investigate a key aspect of social interactions viz., interpersonal comparisons. Thus, our analysis complements the analysis of Akerlof and Kranton. 


\section{CONCLUSION}

The pursuit of migration is an extreme example of the severing of ties with, or a distancing from, one's friends and home. The "failure" of migrants to assimilate cannot plausibly be attributed merely to an urge to stay close to their friends, or they might not have migrated to begin with. Non-assimilation arises from a fear of enhanced relative deprivation if they reduce their distance from the natives as a reference group. Fundamentally, migration is a change of those with whom people associate. But it would be wrong to infer that a change of associates crowds out a change of behavior, given the associates. Through their actions, migrants can elect to associate more with some groups, less with others. However, when actions to keep in check the weight accorded to the rich natives as a reference group are not viable, the very choice of migration destination could be affected in an unexpected way: a country that is not so rich could be preferable to a country that is rich; migrants will protect themselves from an unfavorable comparison by not migrating to where the comparison, when unavoidable, would be highly unfavorable. Relatedly, the variance in the assimilation effort of migrants across host countries could arise from the variance in the income distance with the natives: the richer the natives, the weaker the effort to assimilate, other things held the same.

There is a fast growing literature documenting the importance of intra-group comparisons in the determination of happiness (cf. the selection in Bruni and Porta, 2005). Inter alia, the evidence suggests that very often people tend to be quite happy, except when they live in situations or settings in which they are brought, or made to compare themselves with a group that is much better off. The new literature is somewhat shy of analyzing the actions people can take in order to shield themselves from depressing exposure. We have sought to help fill this void. 


\section{REFERENCES}

Agarwal, Bina and Alessandro Vercelli (2005). Psychology, Rationality and Economic Behavior. Houndmills: Palgrave Macmillan.

Akerlof, George A. (1997). Social Distance and Social Decisions, Econometrica. 65: 1005-1027.

Akerlof, George A. and Rachel E. Kranton (2000). Economics and Identity, Quarterly Journal of Economics. 115: 715-753.

Austen-Smith, David and Roland G. Fryer Jr. (2005). An Economic Analysis of 'Acting White', Quarterly Journal of Economics. 120: 551-583.

Borjas, George J. (1995). Assimilation and Changes in Cohort Quality Revisited: What Happened to Immigrant Earnings in the 1980s?, Journal of Labor Economics. 13: 201-45.

Borjas, George J., Stephen G. Bronars and Stephen J. Trejo (1992). Assimilation and the Earnings of Young Internal Migrants, The Review of Economics and Statistics. 74: 170-75.

Bruni, Luigino and Pier Luigi Porta (2005). Economics and Happiness: Framing the Analysis. Oxford: Oxford University Press.

Chiswick, Barry R. (2005). The Economics of Immigration: Selected Papers of Barry R. Chiswick. Cheltenham, U.K.: Elgar.

Cutler, David M., Edward L. Glaeser and Jacob L. Vigdor (2005). Is the Melting Pot Still Hot? Explaining the Resurgence of Immigrant Segregation, Harvard University, Cambridge MA: Harvard Institute of Economic Research, Discussion Paper. No. 2071.

Eibner, Christine and William N. Evans (2005). Relative Deprivation, Poor Health Habits, and Mortality, Journal of Human Resources. 40: 591-620.

Friedman, Jonathan and Shalini Randeria (2004). Worlds on the Move: Globalization, Migration and Cultural Security. London: I.B. Tauris.

Lazear, Edward P. (1999). Culture and Language, Journal of Political Economy. 107: S95-126.

Luttmer, Erzo F. P. (2005). Neighbors as Negatives: Relative Earnings and Well-Being, Quarterly Journal of Economics. 120: 963-1002. 
Stark, Oded (1993). The Migration of Labor. Oxford and Cambridge, MA: Blackwell.

Stark, Oded and You Qiang Wang (2005). Towards a Theory of Self-Segregation as a Response to Relative Deprivation: Steady-State Outcomes and Social Welfare, in: Luigino Bruni and Pier Luigi Porta (eds.), Economics and Happiness: Framing the Analysis. Oxford: Oxford University Press: 223-242.

Veblen, Thorstein (1924). The Theory of the Leisure Class: An Economic Study of Institutions. London: Allen \& Unwin.

Walker, Iain and Heather J. Smith (2002). Relative Deprivation: Specification, Development, and Integration. Cambridge: Cambridge University Press.

Yitzhaki, Shlomo (1979). Relative Deprivation and the Gini Coefficient, Quarterly Journal of Economics. 93: 321-24.

\section{SUMMARY}

The pursuit of migration is an extreme example of the severing of ties with, or a distancing from, one's friends and home. The "failure" of migrants to assimilate cannot plausibly be attributed merely to an urge to stay close to their friends, or they might not have migrated to begin with. Non-assimilation arises from a fear of enhanced relative deprivation if they reduce their distance from the natives as a reference group. Fundamentally, migration is a change of those with whom people associate. But it would be wrong to infer that a change of associates crowds out a change of behavior, given the associates. Through their actions, migrants can elect to associate more with some groups, less with others. However, when actions to keep in check the weight accorded to the rich natives as a reference group are not viable, the very choice of migration destination could be affected in an unexpected way: a country that is not so rich could be preferable to a country that is rich; migrants will protect themselves from an unfavorable comparison by not migrating to where the comparison, when unavoidable, would be highly unfavorable. Relatedly, the variance in the assimilation effort of migrants across host countries could arise from the variance in the income distance with the natives: the richer the natives, the weaker the effort to assimilate, other things held the same. 